\title{
Inspeção Automática de Defeitos em Madeiras de Pinus usando Visão Computacional
}

\author{
Luiz E. S. Oliveira ${ }^{1}$ \\ Paulo R. Cavalin ${ }^{2}$ \\ Alceu S. Britto Jr ${ }^{1,3}$ \\ Alessandro L. Koerich ${ }^{1}$
}

\begin{abstract}
Resumo: Este artigo apresenta um método completo para a detecção de defeitos em tábuas de madeira de Pinus através de técnicas de Visão Computacional. As imagens dos lados da tábua de madeira são adquiridas com câmeras tipo line scan e processadas para extração de características baseadas na informação cor e em propriedades de textura: suavidade, aspereza e regularidade. Um subconjunto destas características, extraído a partir de imagens em níveis de cinza e selecionado com base em algoritmos genéticos multi-objetivos é proposto como alternativa para redução de custos no processo de aquisição de imagens. Dois paradigmas de aprendizagem de máquina diferentes foram utilizados: redes neurais e máquinas de vetor de suporte. Resultados experimentais demonstram que o conjunto de características selecionado a partir de imagens em níveis de cinza atingiu desempenho competitivo para o problema de detecção de defeitos em madeira, quando comparado com conjunto de características que depende de sensor de aquisição de maior custo (line scan colorida) para extração de características baseadas na informação cor.
\end{abstract}

Palavras Chaves: inspeção de madeira, detecção de defeitos em madeira, seleção de características, $S V M, M L P$.

\footnotetext{
${ }^{1}$ Pontifícia Universidade Católica do Paraná, Programa de Pós-Graduação em Informática Aplicada (PPGIa), Curitiba (PR), Brasil, \{alceu, alekoe, soares@ppgia.pucpr.br

${ }^{2}$ École de Technologie Supérieure (ÉTS), Montreal, Canadá, cavalin@ livia.etsmtl.ca

${ }^{3}$ Universidade Estadual de Ponta Grossa (UEPG), Ponta Grossa (PR), Brasil.
} 
Abstract: This paper addresses the issue of detecting defects in Pine wood using features extracted from grayscale images. The feature set proposed here is based on the concept of texture and it is computed from the co-occurrence matrices. The features provide measures of properties such as smoothness, coarseness, and regularity. Comparative experiments using a color image based feature set extracted from percentile histograms are carried to demonstrate the efficiency of the proposed feature set. Two different learning paradigms, neural networks and support vector machines, and a feature selection algorithm based on multi-objective genetic algorithms were considered in our experiments. The experimental results show that after feature selection, the grayscale image based feature set achieves very competitive performance for the problem of wood defect detection relative to the color image based features.

Keywords: wood inspection, detection of wood defects, feature selection, SVM, MLP. 


\section{Introdução}

A eliminação de desperdícios e a maximização do valor da matéria-prima têm sido os grandes desafios da indústria madeireira nos últimos anos. Nesta direção, a introdução de novas tecnologias, incluindo-se os Sistemas de Visão Computacional, confere à indústria e seus produtos a qualidade, confiabilidade e competitividade exigidas pelo mercado mundial. Além disso, a adoção de tecnologias que garantam a maximização do aproveitamento deste recurso natural evidencia a preocupação da indústria com a redução de passivos ambientais.

O processo de beneficiamento tem como objetivo maximizar o valor da madeira, podendo ser dividido basicamente em três etapas. Inicialmente a tora é conduzida para a serraria, onde se decide se a mesma será mais valiosa se transformada em tábuas, compensados ou ainda pequenos blocos. Uma vez decidido que seu destino é a transformação em tábuas, retiram-se as placas a partir do tronco, as quais devem ser posteriormente trabalhadas. Este processo requer que alguém decida como realizar o corte maximizando qualidade e quantidade. Cada tábua deve ser examinada e classificada segundo critérios pré-estabelecidos no mercado madeireiro, com base na qualidade da madeira e na presença de defeitos. Em uma etapa final, ainda é possível acrescentar valor ao produto cortando-se as tábuas com o objetivo de se produzir segmentos livres de defeito. Neste ponto, merece destaque especial o processo de otimização do corte, onde modernos equipamentos efetuam o reconhecimento de defeitos com auxílio de um scanner e marcação manual (giz fluorescente). A eliminação dos defeitos das tábuas é controlada automaticamente, classificando os diversos segmentos obtidos, além de quantificar a produção e perdas. A capacidade de produção de tais otimizadoras de corte varia de acordo com a qualidade da madeira sendo processada, podendo atingir $1.200 \mathrm{~m} / \mathrm{h}$ (Figura 1).

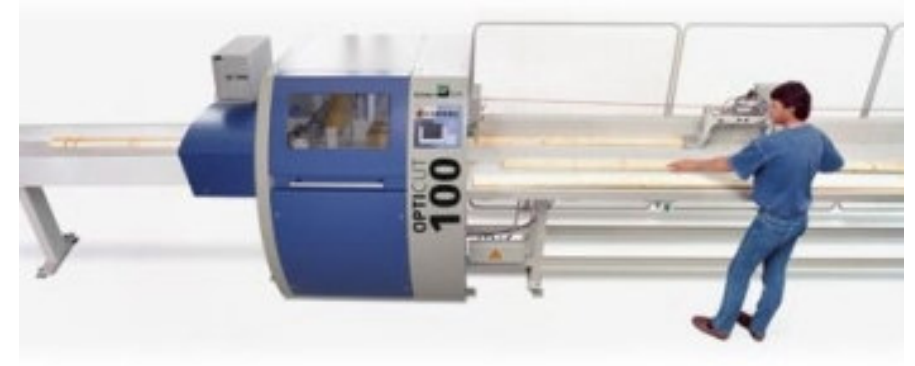

Figura 1. Exemplo de uma máquina de otimização de corte. Operador alimenta a máquina e normalmente delimita com giz fluorescente as regiões com defeitos. 
Contudo, para a grande maioria destas máquinas é necessário um alto nível de interação humana, uma vez que o operador deve identificar e marcar (assinalar) com giz fluorescente os defeitos encontrados na tábua. É sabido que raramente os operadores seguem regras bem definidas, e normalmente baseiam sua decisão na aparência visual da tábua. Um dos problemas claros está na falta de padrão para essa tomada de decisão, pois alguns operadores são menos eficientes que outros na detecção de pequenos defeitos, tais como: pequenas rachaduras e manchas.

A aparência visual de uma tábua é, em geral, descrita por atributos como cor e forma [1]. Com base nesta hipótese, alguns autores têm mostrado, experimentalmente, que a cor é uma informação muito importante dado o seu poder de discriminação em sistemas de inspeção de madeira, especialmente quando o objetivo é a detecção de defeitos [2]-[4].

O foco deste artigo está no principal problema relacionado à construção de uma máquina de otimização de corte de madeira: a detecção de defeitos. O objetivo é propor um método para detectar defeitos em madeira de Pinus, que seja robusto e de baixo custo. Uma vez que uma das restrições é o custo, opta-se por sensores de captura monocromáticos, os quais são consideravelmente mais baratos que sensores coloridos, principalmente quando se necessita de sensores de linha (line scan sensors). Desta forma, um dos desafios deste trabalho está na seleção de um conjunto de características para detecção de defeitos em madeira que seja extraído de imagens em escala de cinza. Na concepção do método proposto foram avaliados dois diferentes paradigmas de aprendizagem de máquina: Máquinas de Vetor de Suporte (MVS) e Redes Neurais (RN). A seleção de características é conduzida por algoritmos genéticos a fim de se evitar qualquer possibilidade de influência de características redundantes e/ou irrelevantes. Um protocolo experimental robusto mostra que as características extraídas a partir de imagens em escala de cinza, quando comparadas àquelas calculadas a partir de imagens coloridas, apresentam desempenho similar para o problema de detecção de defeitos em madeira. Os resultados são comparados ao obtidos por [3], cujo método se baseia em histogramas cumulativos calculados a partir dos canais $R, G$ e $B$ de imagens coloridas.

Este artigo está organizado como segue: A Seção 2 apresenta uma visão geral do método proposto. A Seção 3 descreve os dois conjuntos de características utilizados neste trabalho. A Seção 4 apresenta a metodologia utilizada na seleção de características, e na Seção 5 descreve-se a base de dados utilizada nos experimentos. A Seção 6 relata os experimentos, enquanto a Seção 7 apresenta as conclusões e trabalhos futuros.

\section{Visão Geral do Método}

O método para inspeção automática de defeitos em madeiras é composto por elementos de hardware e software. A Figura 2 apresenta os principais elementos deste método. 


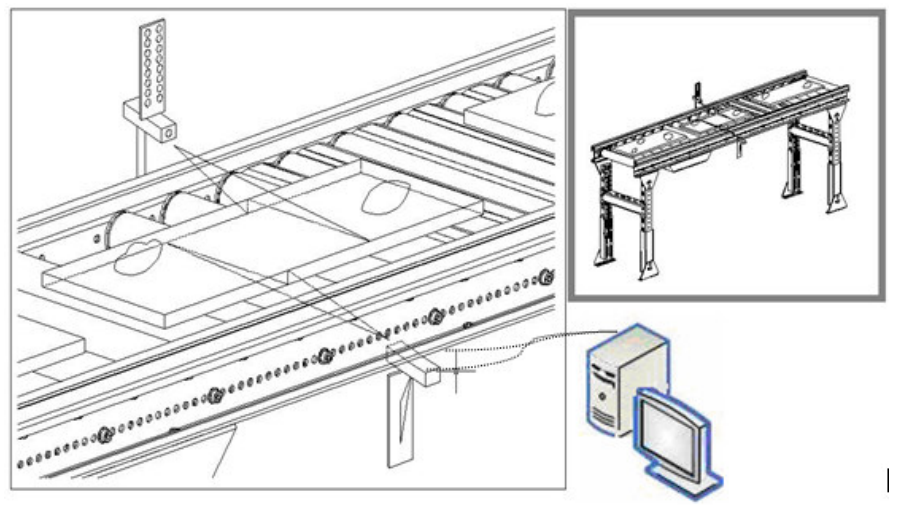

Figura 2. Principais componentes do método proposto: 2 câmeras line scan, computador, iluminação baseada em LEDs e transportador

O processo de inspeção inicia-se com a passagem de uma tábua de madeira por um transportador. Duas câmeras tipo line scan e sistema de iluminação baseado em LEDS permitem a captura de imagens de ambas as faces da madeira de forma sincronizada com a velocidade do transportador. Uma vez adquiridas as imagens completas de ambas as faces da madeira, estas são processadas por um módulo de extração de características baseadas em cor, ou em textura conforme proposto neste artigo. Um processo de seleção de características permite a eliminação de características redundantes ou irrelevantes. As características selecionadas são utilizadas como entrada de um classificador de padrões previamente treinado. O objetivo do classificador é localizar e quantificar defeitos em tábuas de madeira, sem a interação humana, visando reduzir custos relacionados aos processos de otimização de corte e/ou classificação da madeira de acordo com padrões pré-estabelecidos. Além das informações sobre os possíveis defeitos presentes na madeira são calculadas informações sobre a dimensão das tábuas processadas, não reportadas neste artigo por considerarmos não relevantes. $\mathrm{O}$ coração do método proposto está na seleção e extração de características, bem como no treinamento do classificador utilizado para detecção dos defeitos em madeira. Nas próximas seções dá-se ênfase a estes módulos.

\section{Conjuntos de Características}

Esta seção descreve os dois conjuntos distintos de características usados neste trabalho. O conjunto de características baseadas em cor leva em consideração histogramas de percentis calculados a partir dos canais de cor Red, Green e Blue do sistema de cor RGB, enquanto o conjunto de características em escala de cinza é extraído a partir de matrizes de co-ocorrência [5]. 


\subsection{Características baseadas em cor}

Os histogramas cumulativos extraídos a partir dos canais de cor R, G e B são simples, mas muito discriminativos, conforme foi comprovado experimentalmente neste trabalho. Alguns percentis são selecionados e os valores de intensidade relacionados são utilizados como primitivas. Considere $C_{k}(i)=\sum_{j=1}^{i} H_{k}(j)$ como o histograma cumulativo da intensidade $i$ no canal $k$, sendo $H_{k}(j)$ o histograma para o valor de intensidade $j$ no canal $k$, e $N_{k}$ o número de canais do sistema de cor em questão (RGB), o valor da característica para o percentil $y$ é:

$$
F_{k}(y)=i, \quad \text { naqual } \quad C_{k}(i) \approx y, \quad 1 \leq i \leq N_{k}
$$

Devido a mudanças de intensidade de características baseadas em percentil puro, um esquema de calibração de intensidade é utilizado. Características invariantes ao deslocamento e largura do histograma podem ser obtidas calculando-se a diferença de dois valores consecutivos de percentil, normalizada pela diferença entre o percentil máximo e mínimo do histograma:

$$
f=\frac{F_{k}\left(y_{1}\right)-F_{k}\left(y_{2}\right)}{F_{k}(100 \%)-F_{k}(0 \%)}
$$

Uma maneira de selecionar as características a partir do percentil é considerar porções iguais em uma faixa entre os valores de máximo e mínimo. Como imagens de madeira podem apresentar algum ruído, é razoável selecionar o valor de máximo e mínimo como $95 \%$ e $5 \%$, respectivamente, bem como dividir a faixa de interesse (90\%) em pedaços iguais. Para os experimentos realizados o vetor de características é obtido a partir de 10 características de percentis para cada canal do sistema de cor. Logo, o número de características neste primeiro vetor é de 30 (10 x 3).

\subsection{Características baseadas em escala de cinza}

Uma abordagem interessante para descrever uma região consiste na quantificação de informações oriundas de sua textura. A análise da textura fornece, intuitivamente, medidas que representam propriedades como suavidade, aspereza e regularidade. As características em escala de cinza propostas para a detecção de defeitos em madeira têm como base a textura e são calculadas a partir da matriz de co-ocorrência. Tais propriedades são medidas estatísticas extraídas a partir da matriz que representa a relação entre pixels dentro de uma região.

A matriz de co-ocorrência ( $M C$ ) é a probabilidade conjunta da ocorrência do nível de cinza $i$ e $j$ dentro de uma relação espacial definida na imagem. A relação espacial é 
definida em termos de uma distância $d$ e de um ângulo $\theta$. Assumindo que $N_{g}$ é a profundidade da escala de cinza e $p(i, j)$ é a probabilidade da co-ocorrência dos níveis de cinza $i$ e $j$ observando-se pixels consecutivos a uma distância $d$ e ângulo $\theta$, as seguintes medidas derivadas de $M C$ foram escolhidas:

$$
\begin{array}{r}
f_{1}=\text { Contraste }=\sum_{i=1}^{N_{g}} \sum_{j=1}^{N_{g}}(i-j)^{2} p(i, j) \\
f_{2}=\text { Energia }=\sum_{i=1}^{N_{g}} \sum_{j=1}^{N_{g}}(p(i, j))^{2} \\
f_{3}=\text { Entropia }=-\sum_{i=1}^{N_{g}} \sum_{j=1}^{N_{g}} p(i, j) \log (p(i, j)) \\
f_{4}=\text { Correlação }=\sum_{i=1}^{N g} \sum_{j=1}^{N g} \frac{p(i, j)-\mu_{x} \mu_{y}}{\sigma_{x}^{2} \sigma_{y}^{2}}
\end{array}
$$

nas quais

$$
\begin{aligned}
& \mu_{x}=\sum_{i=1}^{N_{g}} i p_{x}(i), \quad p_{x}(i)=\sum_{j=1}^{N_{g}} p(i, j), \quad \sigma_{x}^{2}=\sum_{i=1}^{N_{g}}\left(i-\mu_{x}\right)^{2} p_{x}(i) \mathrm{e} \\
& \mu_{y}=\sum_{i=1}^{N_{g}} i p_{y}(i), \quad p_{y}(i)=\sum_{j=1}^{N_{g}} p(i, j), \quad \sigma_{y}^{2}=\sum_{i=1}^{N_{g}}\left(i-\mu_{y}\right)^{2} p_{y}(i) .
\end{aligned}
$$

As medidas acima foram extraídas usando-se diferentes distâncias $d$ e ângulos $\theta$ para cálculo de $M C$. Para cada $M C, 4$ medidas são adicionadas ao vetor de características $\bar{f}$. Uma medida adicional diz respeito ao nível de cinza médio da imagem. Portanto, o tamanho do vetor $\bar{f}$ é $4 \times N_{d \theta}+1$, sendo $N_{d \theta}$ o número de combinações entre $d$ e $\theta$. Nos experimentos foram avaliados diferentes valores para $d$ e $\theta$. A melhor combinação encontrada foi $d=1$ e $\theta=[0,90,180,270]$, o que define um vetor com 17 características.

\section{Seleção de Características}

Um fator importante na construção de um classificador é a seleção das características mais discriminativas. Em muitas aplicações é comum encontrar problemas envolvendo centenas de características. Contudo, observa-se que a inclusão de novas características nem sempre se traduz em melhores resultados, e em alguns casos, pode até representar prejuízo ao 
desempenho de um classificador [6]. Além disso, a escolha das características a serem utilizadas na representação de um padrão de interesse afeta vários fatores relacionados ao problema de reconhecimento, tais como: precisão, tempo requerido para o aprendizado, e número de amostras necessárias.

Este aparente paradoxo se apresenta como um problema de seleção de características para projeto automático de um classificador. Tal problema se refere à tarefa de identificar e selecionar em subconjunto efetivo de características para representar padrões a partir de um conjunto maior de características, freqüentemente redundantes ou mesmo irrelevantes.

A seleção de características não é um problema trivial, pois raramente estas são totalmente independentes. Podem existir redundâncias, ou seja, características correlacionadas, caso em que não devem ser consideras juntas na modelagem, e interdependências, quando duas ou mais características contêm informação importante entre si que pode se tornar obscura se alguma dessas características não for considerada na modelagem.

Na prática, a seleção de características consiste em uma função de otimização multicritérios, na qual busca-se minimizar o número de características e maximizar desempenho do classificador. É possível observar na literatura que algoritmos genéticos multi-objetivo têm se mostrado uma abordagem atrativa para este tipo de problema, uma vez que estes são em geral muito eficientes para buscas globais em grandes espaços não-lineares, do qual se tem pouco entendimento. Além de poderem trabalhar com vários objetivos de maneira inteligente. Com base nestas premissas, utilizou-se a estratégia proposta em [7] para realizar a seleção de características, com base em um poderoso algoritmo genético multi-objetivo chamado NSGA (acrônimo em inglês para Non-Dominated Sorting Algorithm) [8].

Diferentemente de um algoritmo genético simples, o NSGA produz um conjunto de soluções potenciais conhecido como solução Pareto-optimal. Isto permite ao usuário avaliar diferentes cenários para os objetivos que estão sendo otimizados. Uma maneira muito interessante de se definir uma solução é considerar uma base de validação independente para evitar uma solução tendenciosa ou superestimada. Para maiores detalhes favor consultar [7].

No NSGA, um método de seleção baseado em ranqueamento é utilizado para enfatizar pontos bons e uma estratégia é usada para manter subpopulações estáveis de pontos bons, denominadas nichos. O NSGA difere de algoritmos genéticos simples somente na maneira que o operador de seleção trabalha. $O$ cruzamento e a mutação permanecem como usual. Antes de a seleção ser realizada, a população é ranqueada com base na não-dominação de cada indivíduo. Os indivíduos não-dominados, presentes na população, são inicialmente identificados na população corrente. Então, assume-se que todos estes indivíduos constituem o primeiro fronte não-dominado na população e recebem um alto valor-objetivo (fitness). $\mathrm{O}$ mesmo valor-objetivo é atribuído para dar um potencial reprodutivo igual para todos estes indivíduos não-dominados.

Para manter a diversidade da população, estes indivíduos classificados são então compartilhados com seus valores-objetivo. O compartilhamento é realizado através da 
operação de seleção usando valores-objetivo degradados, os quais foram obtidos dividindose o valor-objetivo original de um individuo por uma quantidade proporcional ao número de indivíduos ao seu redor. Em seguida, a população é reproduzida de acordo com os valoresobjetivo. Uma vez que indivíduos no primeiro fronte têm valor-objetivo máximo, eles são mais copiados que o resto da população. A eficiência do NSGA está na maneira com que múltiplos objetivos são reduzidos a uma função objetivo usando procedimento de ordenação não-dominada (ver Figura 3).

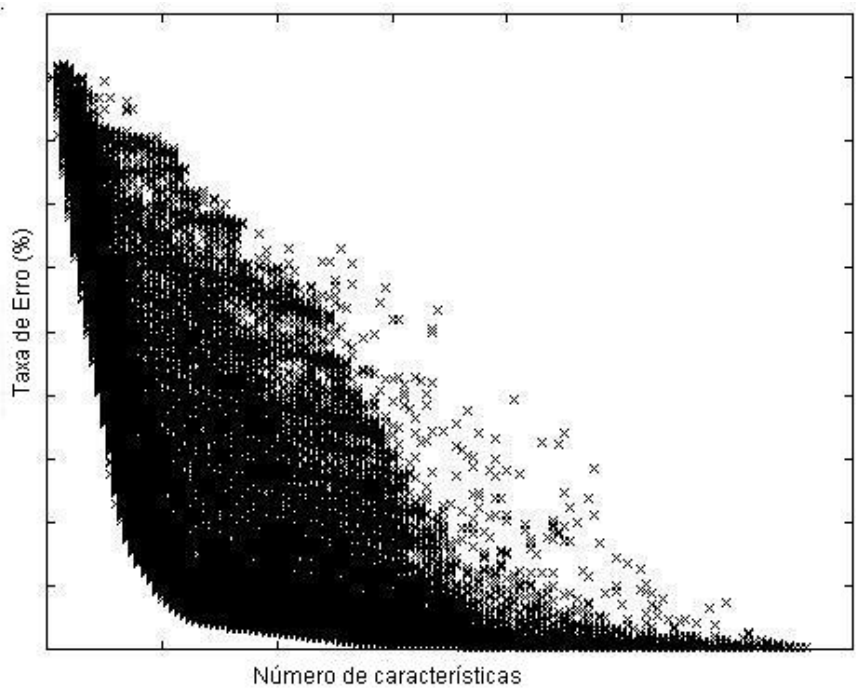

Figura 3. Exemplo da evolução no plano objetivo

No final, o algoritmo produz um conjunto de soluções potenciais que podem ser escolhidas por um mecanismo de decisão. Como mencionado anteriormente, um mecanismo eficiente consiste na avaliação das soluções potencias com base em um conjunto de validação independente, evitando assim soluções tendenciosas ou superestimadas. A Figura 4 apresenta um fronte clássico, tipo Pareto-optimal, para o problema de seleção de características abordado neste trabalho.

Analisando somente o fronte Pareto, o melhor compromisso entre o número de características e a taxa de erro é representada pela solução $S_{1}$. Contudo, analisando a curva de validação, observa-se que tal solução não representa uma boa generalização quando se utiliza uma base de amostras desconhecidas. É possível observar ainda, que o compromisso entre desempenho e complexidade que apresenta a melhor generalização na base de validação é a solução $S_{2}$. 


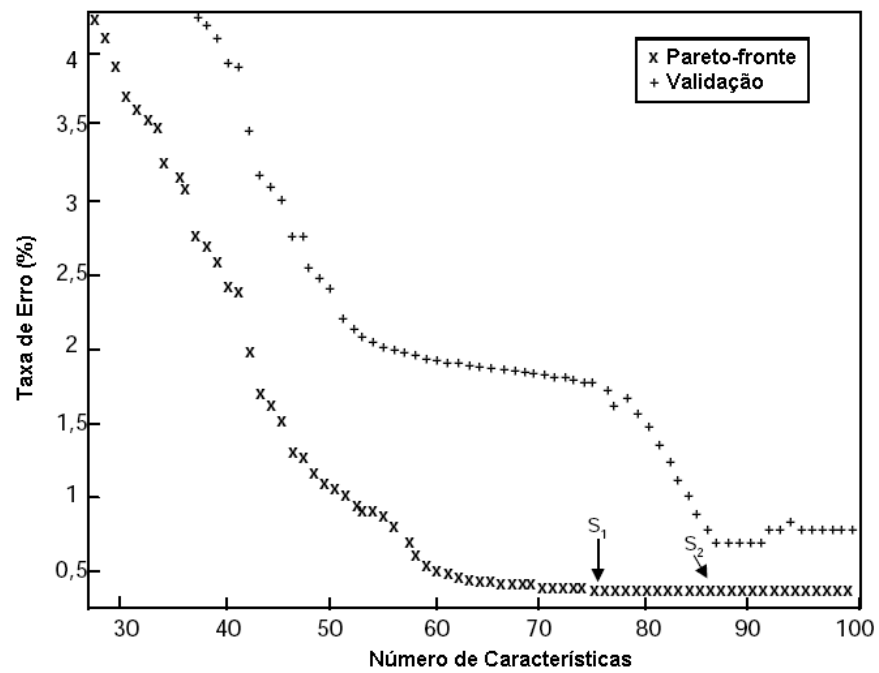

Figura 4. Pareto-optimal produzido pelo NSGA

\section{Treinamento das Características Selecionadas}

A abordagem utilizada toma por base uma segmentação implícita, que significa o particionamento da imagem sem considerar o seu conteúdo, utilizando-se um gride previamente definido. Desta maneira, o particionamento é feito em regiões não-sobrepostas de $32 \times 32$ pixels conforme proposto em [9].

As características são calculadas para cada região e então classificadas nas classes relevantes. No caso de detecção de defeitos, consideram-se duas classes: região boa e região com defeito. Uma interface gráfica foi construída especialmente para rotulação da base de treinamento. Esta interface segmenta a imagem em janelas de 32 × 32 pixels e aquelas contendo algum defeito são manualmente classificadas, por um especialista, como classe 2 (região com defeito). As regiões não marcadas são automaticamente rotuladas como classe 1 (região boa).

A base de imagens considerada neste trabalho é composta por 500 imagens extraídas de tábuas de Pinus. Foram utilizadas 300 imagens para treinamento, 100 para validação e 100 para testes. A base contém vários tipos de defeitos, os quais são apresentados nas Figuras 5 e 6 . A maioria dos defeitos está relacionada à presença de nós nas tábuas, os quais podem ser inicialmente classificados conforme sua consistência em firmes, secos (ou soltos), encaixados, deteriorados ou buracos de nós. Em uma segunda classificação, os nós ainda podem ser classificados conforme sua forma e localização na tábua em: normal, borda, folha e chifre. 
A Figura 7 mostra exemplo de uma imagem segmentada em regiões de 32 x 32 pixels. Os blocos considerados com defeito estão em destaque.

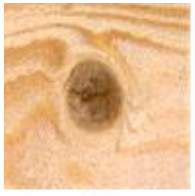

(a)

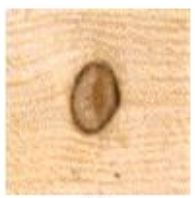

(e)

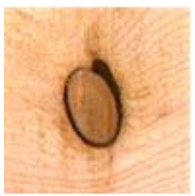

(i)

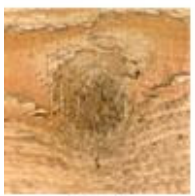

(m)

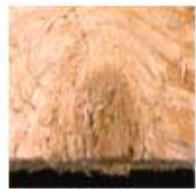

(b)

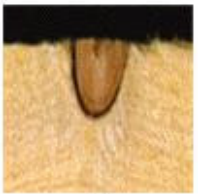

(f)

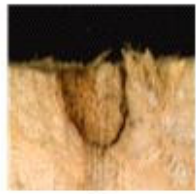

(j)

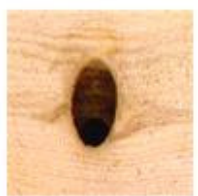

(n)

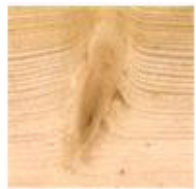

(c)

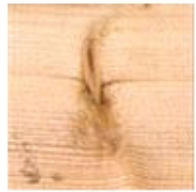

(g)

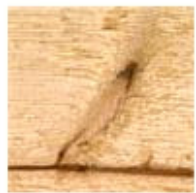

(k)

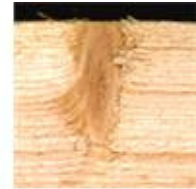

(d)

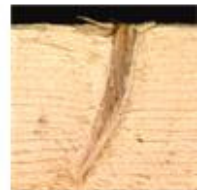

(h)

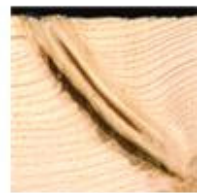

(I)

Figura 5. Tipos diferentes de nós encontrados na base: (a-d) nós firmes (normal, borda, folha e chifre), (e-h) nós secos ou soltos (normal, borda, folha e chifre), (i-1) nós encaixados (normal, borda, folha e chifre), (m) nó deteriorado, (n) buraco de nó.

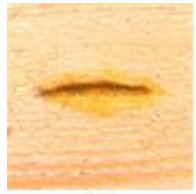

(a)

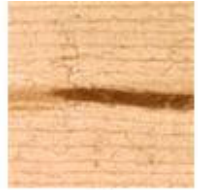

(b)

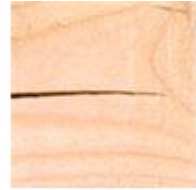

(c)

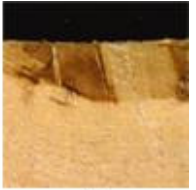

(d)

Figura 6. Outros defeitos: (a) bolsa de resina, (b) mancha marrom, (c) racha e (d) lascada nas bordas. 
As imagens foram coletadas usando uma câmera CCD Samsung com precisão de 8bits para cada canal de cor. Isto permite uma comparação entre as características baseadas em cor e em escala de cinza. Para criar a imagem em escala de cinza, foi utilizada a equação (7) para determinação da luminância.

$$
G R A Y=[0,299 ; 0,587 ; 0,114] \times[R, G, B]^{T}
$$

\section{Resultados Experimentais}

Durante os experimentos foram avaliados dois diferentes paradigmas de aprendizagem de máquina: Redes Neurais (RN) e Máquinas de Vetor de Suporte (SVM, do inglês Support Vector Machines). A rede neural utilizada é uma MLP (do inglês, Multi-Layer Perceptron) treinada com o gradiente descendente aplicado a uma função de soma dos erros quadráticos. A função de transferência empregada é a função sigmoid padrão. O desempenho em termos de generalização é monitorado através da validação e os parâmetros das redes foram determinados empiricamente.

O outro algoritmo de aprendizado considerado é uma Máquina de Vetor de Suporte [10]. Esta abordagem tem recebido atenção especial da comunidade de reconhecimento de padrões e aprendizagem de máquina devido a sua habilidade em generalizar mesmo quando aplicadas em espaços de alta dimensionalidade em situações onde existam poucos exemplares para treinamento. Nos experimentos realizados utilizou-se o pacote LIBSVM [11]. Os parâmetros do classificador SVM foram selecionados utilizando-se uma ferramenta de pesquisa em gride disponível no LIBSVM.

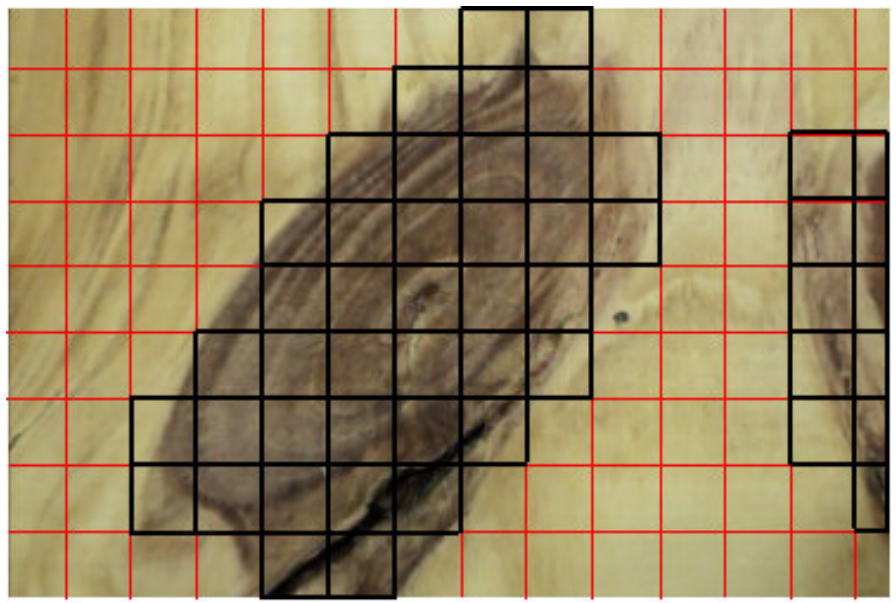

Figura 7. Exemplo do janelamento ( 32 x 32 pixels) para um segmento de tábua de Pinus, onde as regiões com defeito estão em destaque. 
O NSGA utilizado para seleção de características baseia-se em representação em bit, cruzamento uni-ponto, mutação bit-flip, e seleção via método roleta (com elitismo). Os seguintes parâmetros de configuração foram utilizados: tamanho da população $=128$, número de gerações $=1000$, probabilidade de cruzamento $=0,8$, probabilidade de mutação $=$ 0,007 , e distância de nichos $\left(\sigma_{\text {share }}\right)=0,5$.

No primeiro experimento foram considerados os vetores de características originais, i.e., sem seleção de características. As duas primeiras colunas da Tabela I apresentam estes resultados. Observa-se que a abordagem baseada em SVM detectou corretamente os defeitos em 98.7\% dos casos.

Tabela I - Detecção de defeitos: desempenho dos classificadores para o conjunto de teste, antes e depois da seleção de características (SC)

\begin{tabular}{|l|l|l|l|l|}
\hline Características & \multicolumn{4}{|c|}{ Taxas de Acerto em \% } \\
\hline & \multicolumn{2}{|c|}{ Antes SC } & \multicolumn{2}{c|}{ Depois SC } \\
\hline & MLP & SVM & MLP & SVM \\
\hline Cor & 98.0 & 98.7 & 98.1 & 98.7 \\
\hline Escala de cinza & 95.5 & 95.8 & 97.5 & 98.0 \\
\hline
\end{tabular}

Ainda é possível observar que as características baseadas em cor apresentam resultados melhores que as características baseadas em escala de cinza para ambos os modelos.

Por outro lado, ainda é possível observar que depois da seleção de características, ambos os classificadores SVM e MLP treinados com características baseadas em escala de cinza alcançaram desempenho similar àqueles treinados com características baseadas em cor. Em termos de número de características, em ambos os casos, houve uma redução significativa. Os conjuntos de características baseadas em cor e em escala de cinza tiveram 21 e 8 características removidas, respectivamente. Isto confirma a importância da seleção de características em qualquer tipo de sistema de reconhecimento de padrões e deixa claro que em ambos os conjuntos iniciais de características existem características mutuamente redundantes ou mesmo irrelevantes.

A análise dos resultados demonstra que o método é capaz de detectar pequenos defeitos tais como rachaduras e manchas. A Fig. 8 mostra um exemplo da classificação de tábua de Pinus. É possível observar que o defeito foi detectado, mas algumas regiões de madeira boa foram classificadas erroneamente como defeito. Este tipo de erro se deve ao contraste dentro da região classificada. Contudo, este tipo de confusão pode ser eliminada através de um estágio de pós-processamento baseado em binarização conforme proposto em [3]. 


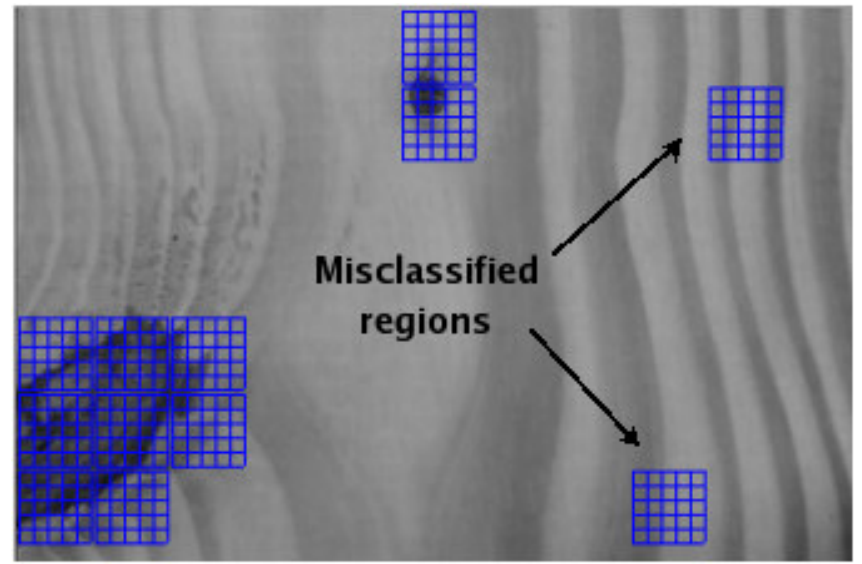

Figura 8. Exemplo de regiões classificadas erroneamente.

\section{Conclusões e Trabalhos Futuros}

Este artigo abordou a detecção de defeitos em madeira através do uso de técnicas de Visão Computacional e Reconhecimento de Padrões. O objetivo foi construir um algoritmo robusto e de baixo custo a fim de aplicar em uma máquina para otimização de corte de madeiras. Conforme descrito anteriormente, a redução de custo pode ser obtida no sensor de aquisição, com a escolha de características em escala de cinza ao invés de coloridas.

Demonstrou-se, experimentalmente, que é possível atingir resultados similares aos obtidos com características coloridas usando apenas informações de textura obtidas de imagens em escala de cinza. É importante ressaltar a contribuição da seleção de características em todo o processo. Em ambos os espaços (cor e escala de cinza) observou-se a redução de características redundantes e/ou irrelevantes. Ainda, no caso do conjunto de características baseado em escala de cinza, observou-se um ganho de desempenho com a redução das características. Como trabalho futuro, planeja-se avaliar o desempenho destas características para a categorização de madeiras.

\section{Referências}

[1] Kauppinen H. "Development of a Color Machine Vision Method for Wood Surface Inspection”. PhD thesis, Oulu University Library, 1999.

[2] Radovan S.; George P.; Panagiotis M.; Manos G.; Robert A., and Igor D.. "An approach for automated inspection of wood boards". In Procs of the International Conference on Image Processing, pp. 798-801, 2001. 
[3] Kauppinen H.. "A two stage defect recognition method for parquet slab grading". In Procs of the International Conference on Pattern Recognition, pp. 803-806, 2000.

[4] Brunner C.C.; Maristany A. G.; Butler D. A.; Vanleeuwen D., and Funck J.. W. "An evaluation of color spaces for detecting defects in Douglas-fir veener". Industrial Metrology, pp. 169-184, 1992.

[5] Aria E. H.; Saradjian M. R.; Amini J. and Lucas C. "Generalized cooccurrence matrix to classify irs-1d images using neural network". In Proceedings of XXth ISPRS Congress, Instanbul, Turkey, July, 2004.

[6] Trunk G. V. “A problem of dimensionality: A simple example”. IEEE Trans.on Pattern Analysis and Machine Intelligence, 1(3):306-307, 1979.

[7] Oliveira L. S.; Sabourin R.; Bortolozzi F., and Suen C. Y. "A methodology for feature selection using multi-objective genetic algorithms for handwritten digit string recognition". International Journal of Pattern Recognition and Artificial Intelligence, 17(6):903-930, 2003.

[8] Deb K. Multi-Objective “Optimization using Evolutionary Algorithms". John Wiley and Sons Ltd, 2nd edition, April, 2002.

[9] Sobey P. J. and Semple E. C. "Detection and sizing visual features in wood using tonal measures and a classification algorithm". Pattern Recogntion, 22:367-380, 1989.

[10] Vapnik V. “Statistical Learning Theory”. John Wiley and Sons, 1998.

[11] Chang C. C. and Lin C-J. LIBSVM: a library for support vector machines. Software available at http://www.csie.ntu.edu.tw/ cjlin/libsvm, 2001. 